\title{
Opinião de gestores sobre trabalhadores mais velhos no contexto organizacional
}

\author{
Management opinion about older workers in the organizational contexto \\ Opinión de la dirección sobre los trabajadores mayores en el contexto organizativo
}

Thais da Silva Pires

ORCID: https://orcid.org/0000-0001-6100-0141 Universidade Estadual de Londrina, Brasil E-mail: Thaiss_pires@hotmail.com

Luiz Fernando da Silva

ORCID: https://orcid.org/0000-0001-7477-1324 Universidade Estadual de Londrina, Brasil

E-mail: luiz_fernando.silva@hotmail.com

Mariana Angela Rossaneis

ORCID: https://orcid.org/0000-0002-8607-0020

Universidade Estadual de Londrina, Brasil

E-mail: marianarossaneis@gmail.com

Maria do Carmmo Fernandez Haddad

ORCID: https://orcid.org/0000-0001-7564-8563

Universidade Estadual de Londrina, Brasil E-mail: carmohaddad@gmail.com

Desirée Ariane Modos Figueira

ORCID: https://orcid.org/0000-0003-1097-3829

Universidade Estadual de Londrina, Brasil E-mail: desireefigueira@hotmail.com

Raquel Gvozd Costa

ORCID: https://orcid.org/0000-0002-5816-8215

Universidade Estadual de Londrina, Brasil E-mail: raquelgvozd@yahoo.com.br

\begin{abstract}
Resumo
Objetivo: Identificar a opinião de gestores sobre trabalhadores mais velhos no contexto organizacional. Método: Estudo transversal, de abordagem quantitativa, realizado com 176 gestores de uma instituição pública. Os dados foram analisados por estatística descritiva, razão de prevalência, intervalo de confiança e p-valor. Resultados: Os gestores do sexo feminino consideraram o envelhecimento da força de trabalho como um problema $(\mathrm{RP}=0,872 / \mathrm{IC}=0,788-0,967 / \mathrm{p}=0,009)$. Os gestores mais velhos afirmaram que o aumento na média de idade do quadro de pessoal impactaria na imagem organizacional $(\mathrm{RP}=0,888 / \mathrm{IC}=0,801-0,985 / \mathrm{p}=0,024)$. Os jovens responderam que o impacto seria em absenteísmo $(\mathrm{RP}=1,148 / \mathrm{IC}=1,045-1,261 / \mathrm{p}=0,004)$. As características favoráveis presentes nos trabalhadores, na visão dos gestores, são produtividade, habilidades sociais, confiabilidade e lealdade. Conclusão: Evidenciou-se percepções diversificadas e discordantes dos gestores sobre os trabalhadores mais velhos. Apesar de reconhecer as características positivas, os gestores ainda relacionam o envelhecimento a problemas, aumento de conflitos e alteração na base de conhecimento da instituição.
\end{abstract}

Palavras-chave: Ageísmo; Envelhecimento; Organização e administração; Trabalhadores; Recursos humanos.

\begin{abstract}
Objetive: To identify the opinion of managers about older workers in the organizational context. Method: Crosssectional study with a quantitative approach, carried out with 176 managers from a public institution. The data were analyzed using descriptive statistics, prevalence ratio, confidence interval and p-value. Results: Female managers considered the aging of the workforce as a problem $(\mathrm{PR}=0.872 / \mathrm{CI}=0.788-0.967 / \mathrm{p}=0.009)$. The older managers stated that the increase in the average age of the workforce would impact the organizational image $(\mathrm{PR}=0.888 / \mathrm{CI}=$ $0.801-0.985 / \mathrm{p}=0.024)$. The youth responded that the impact would be absenteeism $(\mathrm{PR}=1.148 / \mathrm{CI}=1.045-1.261 /$ $\mathrm{p}=0.004)$. The favorable characteristics present in workers, in the view of managers, are productivity, social skills, reliability and loyalty. Conclusion: Diversified and discordant perceptions of managers about older workers were evidenced. Despite recognizing the positive characteristics, managers still relate aging to problems, increased conflicts and changes in the institution's knowledge base.
\end{abstract}

Keywords: Ageism; Aging; Organization and administration; Workers; Human resources. 


\begin{abstract}
Resumen
Objetivo: Identificar la opinión de los directivos sobre los trabajadores mayores en el contexto organizacional. Método: Estudio transversal con enfoque cuantitativo, realizado con 176 gerentes de una institución pública. Los datos se analizaron mediante estadística descriptiva, razón de prevalencia, intervalo de confianza y valor p. Resultados: Las mujeres directivas consideraron el envejecimiento de la población activa como un problema $(\mathrm{RP}=$ 0,872 / IC $=0,788-0,967 / \mathrm{p}=0,009)$. Los gerentes de mayor edad manifestaron que el aumento en la edad promedio de la fuerza laboral impactaría la imagen organizacional $(\mathrm{PR}=0.888 / \mathrm{CI}=0.801-0.985 / \mathrm{p}=0.024)$. Los jóvenes respondieron que el impacto sería el absentismo $(\mathrm{PR}=1,148 / \mathrm{IC}=1,045-1,261 / \mathrm{p}=0,004)$. Las características favorables presentes en los trabajadores, a juicio de los directivos, son la productividad, las habilidades sociales, la fiabilidad y la lealtad. Conclusión: Se evidenciaron percepciones diversificadas y discordantes de los gerentes sobre los trabajadores mayores. A pesar de reconocer las características positivas, los gerentes aún relacionan el envejecimiento con los problemas, el aumento de conflictos y los cambios en la base de conocimientos de la institución.
\end{abstract}

Palabras clave: Edadismo; Envejecimiento; Organización y administración; Trabajadores; Recursos humanos.

\title{
1. Introdução
}

O envelhecimento da população mundial, fenômeno que resulta das baixas taxas de fecundidade, queda nos índices de mortalidade e aumento da expectativa de vida, vem ganhando cada vez mais importância nos países em desenvolvimento (Rocha et al., 2018; Sato, Barros, Jardim, Ratier \& Lancman, 2017). Estima-se que a partir de 2040 a população brasileira será constituída por aproximadamente $57 \%$ de pessoas com idade acima dos 45 anos, um cenário que irá exigir iniciativas de gestão específicas às necessidades desta parcela da população (Marcial, Curado, Oliveira, Cruz Júnior, \& Couto, 2017).

O aumento da inserção dessa população no mercado de trabalho, exige a adaptação do idoso e das organizações (Paolini, 2016). Os atuais processos de trabalho não levam em consideração as perdas da capacidade para o trabalho decorrentes da idade. Assim, mesmo com o aumento de idosos trabalhando, a maior parte das configurações de trabalho atuais ainda não estão preparadas para atendê-los adequadamente (Sato, Barros, Jardim, Ratier \& Lancman, 2017).

Ainda não há um consenso ao definir a idade do trabalhador mais velho, podendo variar de 40 até 75 anos (Silva \& Dal Prá, 2014). A partir dos 60 anos, a perda de algumas capacidades funcionais tende a se acentuar (Sato, Barros, Jardim, Ratier \& Lancman, 2017; Raposo, Leite, Maciel, 2018) e como consequência, esses trabalhadores podem sofrer preconceitos por parte dos empregadores. Este preconceito pode estar relacionado ao dado que aponta que entre os anos 1984 e 2014 , houve crescimento da população masculina brasileira com idade entre 50 a 64 anos, que não trabalha e não é aposentada (Camarano, 2016).

No mercado de trabalho, estereótipos de idade influenciam processos de recrutamento, programas de retenção e desenvolvimento, análise de clima organizacional e saúde ocupacional. A idade constitui um fator limitante para a manutenção e inserção dos trabalhadores, causando desânimo em muitos desempregados mais velhos (da Rocha Siqueira-Brito, França, \& Valentini, 2016); Rego, Vitória, Cunha, Tupinambá, \& Leal, 2017).

No Brasil, caracterizado como preconceito e discriminação relacionados à idade, o ageísmo é um fenômeno partilhado na sociedade, relacionado às experiências sociais e que reflete a imagem, o posicionamento e as relações do idoso na estrutura social (de Oliveira Teixeira, de Souza \& Maia, 2018).

Os gestores são responsáveis pela aproximação das diferentes idades, do redelineamento de recursos humanos e da possível reinserção do trabalhador aposentado. Dessa forma, o modo como eles percebem os trabalhadores mais velhos pode ter um efeito significativo na maneira como lidam com o envelhecimento da força de trabalho (Rego, Vitória, Cunha, Tupinambá, \& Leal, 2017).

Neste contexto, vale ressaltar a importância de estudos que se atenham a este panorama e considerem as consequências do elevado número de profissionais com idade mais avançada que atuam nas organizações, avaliando de que forma estes são vistos pelos gestores. 
A partir dessa problemática, que abrange o envelhecimento do trabalhador segundo a perspectiva do gestor, o objetivo do estudo foi identificar a opinião de gestores sobre os trabalhadores mais velhos.

\section{Metodologia}

Trata-se de um estudo transversal quantitativo. Centrada na objetividade, a pesquisa quantitativa tem a característica de ter os resultados quantificáveis e considera que a realidade só pode ser compreendida com base na análise de dados brutos (Pereira \& Shitsuka, 2018). O estudo foi realizado em uma instituição universitária pública do sul do Brasil. Fundada em 1975, a instituição possui 4.426 servidores e 18.491 alunos que estão distribuídos em 53 cursos de graduação e 261 cursos de pós graduação (Universidade Estadual de Londrina, 2019).

A população do estudo abrangeu 732 servidores que exerciam cargo de gestão na instituição, estes foram identificados por meio de uma lista disponibilizada pelo setor de Recursos Humanos, constando as seguintes variáveis: nome, profissão exercida, cargo, setor de trabalho e endereço eletrônico.

Para o cálculo da amostra considerou-se amostragem estratificada, com alocação proporcional ao número de indivíduos em cada cargo. A amostra resultou em 199 indivíduos, distribuídos entre os cargos de: pró-reitor (03) diretor (16), vice-diretor (03), assessor (20), chefes de divisão e de departamento (60), coordenador (23), administrador (03), encarregado de seção (70) e gerente (01).

A coleta de dados ocorreu no período de março a junho de 2017. O instrumento utilizado foi adaptado de dois questionários, ambos referentes a estudos que tinham o objetivo de identificar as percepções de gestores de Recursos Humanos sobre o envelhecimento nas organizações.

O instrumento "Envelhecimento no contexto organizacional" (França et al., 2014), contém 45 questões, que abordam os seguintes aspectos: dados sobre a organização; empregabilidade; gerência de recursos humanos; trabalhadores mais velhos e envelhecimento; o futuro do mercado de trabalho e as políticas de emprego e dados demográficos. "O envelhecimento nas organizações" (Cepellos \& Tonelli, 2017), contempla 76 questões e é composto por quatro partes: caracterização do respondente; caracterização da empresa; atitudes de gestores de Recursos humanos com relação aos trabalhadores mais velhos e práticas de gestão da idade adotadas pelas empresas.

Os autores autorizaram a utilização e adaptação dos instrumentos para a realidade deste estudo. Foram excluídas dos instrumentos as questões que não eram pertinentes para a realidade em estudo. $\mathrm{O}$ instrumento final passou por processo de validação de conteúdo por um grupo de juízes, composto por cinco docentes da área de gestão em saúde e três doutorandos participantes de um grupo de pesquisa sobre gestão em saúde, obtendo $85 \%$ de percentual de validade de conteúdo.

A versão final do instrumento adaptado contém 28 questões, sendo 17 objetivas e 11 abertas. É composto por três partes, sendo a primeira referente às variáveis caracterizadoras do respondente, como sexo, idade, grau de formação e dados ocupacionais. A segunda diz respeito à gestão de recursos humanos, como o número de trabalhadores que estão sob a gestão do respondente e o percentual de trabalhadores no seu setor que têm 50 anos ou mais. A terceira parte do questionário relaciona-se às percepções do gestor em relação aos trabalhadores mais velhos, como a definição de trabalhador mais velho, quais características se aplicam ao profissional mais velho e benefícios e barreiras em possuir profissionais mais velhos no contexto organizacional.

O questionário foi aplicado por meio de ferramenta on-line e enviado via e-mail a todos os gestores que constavam na lista. Nesta etapa obtiveram-se 66 respostas. Para atingir o número calculado da amostra, optou-se por convidar o gestor selecionado a participar da pesquisa por meio de contato telefônico. Realizou-se uma tentativa de contato telefônico para o 
gestor sorteado. Após esta tentativa, foram obtidas 176 respostas, tendo uma perda amostral de 12\%, devido aos absenteísmos, como férias, licenças e atestados médicos.

Os dados foram tabulados em planilha eletrônica e analisados por meio do programa estatístico Statistical Package for the Social Science (SPSS), versão 20.0. As respostas obtidas por meio de escala de likert, sobre os efeitos do aumento da média de idade do quadro de pessoal, foram categorizadas em baixo e alto impacto, analisando a correlação das variáveis para comparar a opinião dos participantes com diferentes sexos e idades. Também foram realizadas análises inferenciais para identificar diferença nas opiniões dos gestores homens e mulheres e de diferentes idades. Essas análises foram apresentadas com cálculos de razão de prevalência $(R P)$, intervalo de confiança $(I C)$ e p-valor (p), identificado por meio do teste de Wald.

A pesquisa foi aprovada pelo Comitê de Ética em Pesquisa da instituição, sob parecer no 1.543.255.

\section{Resultados}

Dos 176 gestores participantes do estudo, 56\% eram do sexo feminino e $47 \%$ possuíam especialização como grau de formação. A faixa etária variou entre 25 e 67 anos, sendo a média de idade 48,16 anos ( $D P=7,94)$ e mediana de 48,0 anos. A média de tempo na instituição foi de 22,03 anos $(D P=7,94)$ e mediana de 23,0 anos. A média de atuação no cargo foi de 7,19 anos $(D P=6,94)$ e mediana de 5,0 anos.

Em relação à frequência do envelhecimento da força de trabalho ser um problema para a gerência do pessoal, 37\% consideraram algumas vezes, sendo que a prevalência de gestores do sexo feminino que consideraram ser um problema foi maior em comparação aos gestores do sexo masculino ( $R P=0,872$ / IC $=0,788-0,967$ / $p=0,009)$.

Ainda, a opinião de que o aumento da média de idade do quadro de pessoal iria produzir um alto impacto nos conflitos no local de trabalho foi mais prevalente entre as mulheres, em relação aos homens ( $R P=1,133$ / IC=1,029-1,248 / $p=0,011)$, porém elas apresentaram menor prevalência ao considerarem que os efeitos do possível aumento da média de idade ocasionariam alto impacto na base de conhecimento da organização ( $R P=0,899$ / IC=0,819-0,987 / $p=0,026)$.

Os gestores com menos de 50 anos tiveram uma menor prevalência de opinião relacionada ao alto impacto do aumento da média de idade na imagem organizacional ( $R P=0,888$ / IC $=0,801-0,985$ / $\mathrm{p}=0,024)$ em comparação com aqueles com mais de 50 anos. Contudo, esses gestores mais jovens apresentaram maior prevalência da opinião em relação à média de idade elevada ocasionar alto impacto no absenteísmo relacionado as licenças médicas $(R P=1,148$ / IC $=1,045-1,261$ / $p=0,004)$.

A maior parte dos gestores $(63,4 \%)$ acredita que o número de trabalhadores no seu setor irá reduzir nos próximos dois anos e 88,6\% consideram que a idade média dos trabalhadores que irão se aposentar aumentará nos próximos 10 anos.

Dentre as características classificadas pelos gestores, as que mais se aplicam aos trabalhadores mais velhos foram: produtividade $(n=51)$, habilidades sociais $(n=49)$, confiabilidade $(n=48)$ e lealdade $(n=47)$. As que menos se aplicam foram: saúde física e vigor ( $n=59)$, novas habilidades tecnológicas $(n=52)$, desejo de aprender ( $n=49)$ e flexibilidade $(n=47)$.

A Figura 1 apresenta os principais benefícios que os gestores consideram em se ter trabalhadores mais velhos no contexto organizacional. 
Figura 1. Percepções de gestores sobre os principais benefícios em ter trabalhadores mais velhos em uma instituição universitária pública do sul do Brasil.

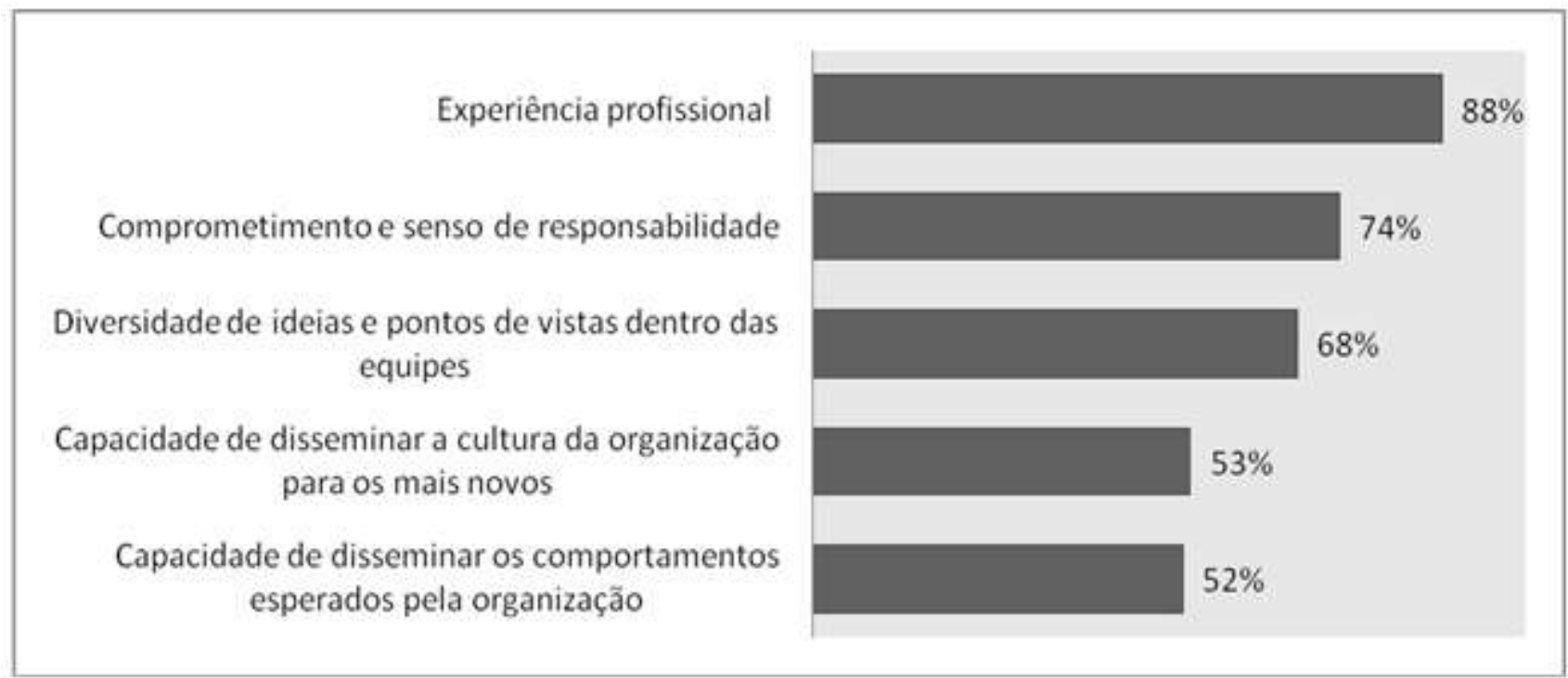

Fonte: Dados obtidos do estudo. Londrina (2017).

Na Figura 2 observam-se as barreiras consideradas pelos gestores em ter trabalhadores mais velhos no contexto organizacional.

Figura 2. Percepções de gestores sobre as principais barreiras em ter trabalhadores mais velhos em uma instituição universitária pública do sul do Brasil.

Acomodação por conta da expectativa da aposentadoria

\section{Dificuldade em lidar com as novas tecnologias}

Dificuldade em se manter atualizado com relação às novas exigências do mercado

Dificuldades de reconhecimento da liderança quando os mais velhos săo liderados pelos mais novos

Falta de flexibilidade e adaptaçăo às mudanças ocorridas na empresa

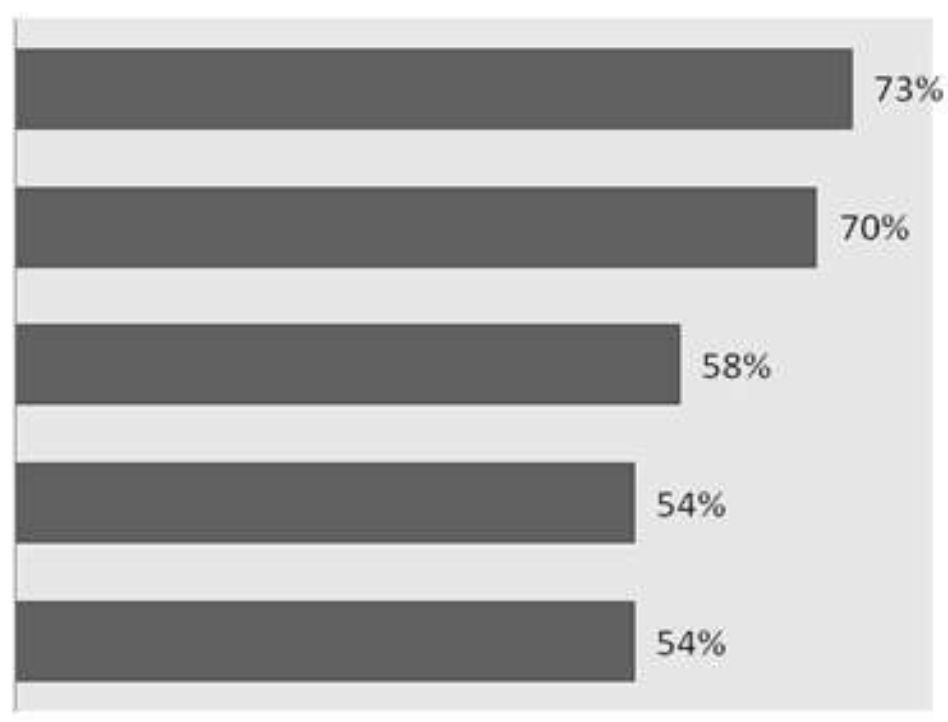

Fonte: Dados obtidos do estudo. Londrina (2017).

Em relação ao Programa de Preparação para Aposentadoria (PPA), 76,9\% dos gestores tinham conhecimento sobre a implantação de um PPA na instituição, sendo que 54,1\% afirmaram que a mesma adota medidas de promoção da saúde para ter uma força de trabalho mais velha e saudável no futuro. 


\section{Discussão}

Os resultados demonstraram que há diferenças nas opiniões dos gestores sobre o trabalhador mais velho, ao comparar as opiniões por sexo e grupos etários dos participantes. As mulheres consideraram ser um problema o envelhecimento da força de trabalho. Elas também avaliaram que os efeitos seriam maiores em conflitos dentro da organização, caso houvesse um aumento na média de idade do quadro de pessoal. Já os homens consideraram que o efeito seria maior na base de conhecimento da organização.

As percepções construídas sobre o envelhecimento são diversas e discordantes, podendo este ser considerado tanto como um processo de ganhos como também de perdas e constituído de acordo com as experiências individuais. O significado de envelhecer está relacionado com estigmas sociais, como a perda da beleza, da força de trabalho e da sanidade, levando a uma percepção negativa acerca do "ser velho" (de Lacerda Araújo, Belo \& Resende, 2016; de Oliveira Teixeira, Marinho, Vasconcelos, \& de Oliveira Martins, 2016).

Um estudo realizado com servidoras de uma instituição pública com idades entre 50 e 68 anos que avaliou a compreensão destas sobre os significados atribuídos ao processo de envelhecer, identificou que há a predominância de uma construção negativa acerca do envelhecimento, principalmente entre as mulheres (Sá \& Wanderbroocke, 2016).

Apesar de reconhecerem as qualidades do trabalhador mais velho e demonstrarem suas atitudes positivas, muitos gestores ainda tomam decisões discriminatórias e continuam a preferir os trabalhadores mais jovens, mesmo quando os mais antigos são descritos como tendo melhores qualidades (de Lacerda Araújo, Belo \& Resende, 2016).

Os indivíduos com idade superior tendem a ter opiniões mais favoráveis acerca dos trabalhadores mais velhos. Por outro lado, os gerentes mais jovens tendem a mostrar atitudes mais negativas em relação aos trabalhadores mais velhos (da Rocha Siqueira-Brito, França, \& Valentini, 2016; de Lacerda Araújo, Belo \& Resende, 2016).

Estudiosos apontam que gestores reconhecem várias características positivas dos trabalhadores mais velhos, por possuírem altos níveis de experiência, conhecimento, sabedoria, lealdade e compromisso para com a organização, além de apresentarem menos absenteísmo e sofrerem menos acidentes de trabalho (da Rocha Siqueira-Brito, França, \& Valentini, 2016; Cepellos \& Tonelli, 2017; França, 2017). Outras percepções positivas que os gestores possuem com relação aos profissionais com mais de 50 anos estão relacionadas a comportamentos como fidelidade à empresa, pontualidade, comprometimento, capacidade de realizar diagnósticos e equilíbrio emocional (da Rocha Siqueira-Brito, França, \& Valentini, 2016; de Lacerda Araújo, Belo \& Resende, 2016; França, 2017).

Uma característica do trabalhador mais velho que foi considerada pela maioria dos gestores foi a produtividade, o que pode ser explicado pelo fato das pessoas que possuem entre 50 e 60 anos, na atualidade, serem mais ativas que os idosos do passado, criando formas eficientes de fazer seu trabalho e permanecendo atuantes por mais tempo, sendo assim, vistas como mais produtivas (da Rocha Siqueira-Brito, França, \& Valentini, 2016; Cepellos \& Tonelli, 2017).

Em relação às barreiras em se ter trabalhadores mais velhos na instituição, os gestores consideraram a acomodação, a dificuldade em lidar com novas tecnologias e em se manter atualizado. A incapacidade de adaptação às mudanças e a dificuldade na introdução de tecnologias na execução do trabalho foram características negativas encontradas em outros estudos (Rego, Vitória, Cunha, Tupinambá \& Leal, 2017; Cepellos \& Tonelli, 2017; França, 2017). Apesar das tecnologias estarem cada vez mais presentes no processo de trabalho das instituições, observa-se que por discriminação, faltam oportunidades para que os trabalhadores mais velhos participem de programas de treinamento, especialmente sobre questões em que eles mais dificuldades, como as novas tecnologias (Rego, Vitória, Cunha, Tupinambá \& Leal, 2017).

Um estudo realizado com gestores de recursos humanos encontrou que dentre as principais percepções negativas dos gestores, estão a incapacidade de realizar trabalho físico pesado, a falta de flexibilidade, dificuldade de adaptação às mudanças, 
preferência por atividades com menos desafios e falta de disposição em receber treinamentos (Raposo, Leite, Maciel, 2018; Rego, Vitória, Cunha, Tupinambá \& Leal, 2017). Outras percepções negativas foram apontadas por estudos, como a maior lentidão no trabalho, desorganização, problemas de relacionamento e tensão no ambiente de trabalho quando o trabalhador mais velho é liderado por profissionais mais jovens (da Rocha Siqueira-Brito, França \& Valentini, 2016; Zaniboni, 2015).

Verificou-se no presente estudo que a saúde e vigor foram características pouco identificadas no trabalhador mais velho. Isso pode ser justificado devido ao processo de diminuição das capacidades físicas, sociais e cognitivas decorrentes do envelhecimento, consideradas indispensáveis para um bom desempenho das funções laborativas, havendo o aumento de doenças. Neste sentido, verifica-se que os principais problemas de saúde de trabalhadores mais velhos consistem em perturbações musculoesqueléticas e psicológicas, sendo a depressão um dos motivos mais comuns para a incapacidade de trabalho (Raposo, Leite, Maciel, 2018).

Por outro lado, destaca-se que a perda de algumas funções pelos trabalhadores mais velhos, que podem prejudicar o desempenho do seu trabalho, pode ser superada por outras características positivas, como o menor índice de absenteísmo, maior satisfação no trabalho e maior comprometimento (Raposo, Leite, Maciel, 2018; Rego, Vitória, Cunha, Tupinambá \& Leal, 2017; Cepellos \& Tonelli, 2017).

A maior parte dos gestores afirmou que o número de trabalhadores no seu setor irá reduzir e que a idade média dos que irão se aposentar aumentará. Isso pode ser explicado devido ao fato de ser uma instituição pública, a qual não há a reposição de trabalhadores regularmente, além de ser constituída por uma grande parcela de servidores que possui maior tempo de trabalho, considerando que a idade média dos participantes foi de 48 anos.

No que se refere às características da amostra estudada, outro resultado que chama atenção é o fato da maioria dos gestores ter o título de especialista. Isso pode estar relacionado com o plano de cargos e carreira que a instituição possui, oferecendo maiores salários aos trabalhadores especializados. A instituição favorece ainda a especialização de seus trabalhadores, com liberações parciais da sua carga horária para este fim. Com o plano de carreira houve uma significativa mudança no perfil dos servidores, relacionada com a vida social e na postura profissional, no qual se observa uma melhoria no desenvolvimento de suas tarefas (de Oliveira Cavalcante \& da Silva, 2017).

Vale destacar o conhecimento dos participantes do estudo sobre a existência de um Programa de Preparação para Aposentadoria na instituição. O Programa, que foi implantado em 2001, é voltado para o trabalhador pré-aposentado e aborda questões relacionadas com o processo de aposentadoria, proporcionando aos servidores momentos reflexivos sobre o projeto de vida voltado ao futuro (Pazzim \& Marin, 2016).

O estudo apresentou como limitação a utilização de um instrumento de coleta de dados em sua maioria objetivo, resultando em respostas prontas e precisas. Dessa forma, ao se discutir percepção, sugere-se a utilização de outros métodos complementares para investigar com mais aprofundamento do envelhecimento do trabalhador nesta perspectiva.

\section{Considerações Finais}

Este estudo evidenciou percepções diversificadas e discordantes de gestores sobre o trabalhador mais velho. Apesar do crescimento do número de trabalhadores envelhecidos e o fato destes estarem mais ativos, o termo envelhecimento ainda é muito relacionado a problemas, aumento de conflitos e alteração na base de conhecimento da instituição.

Embora reconheçam várias características positivas como: alto nível de experiência, lealdade e a pontualidade, os gerentes colocam como barreira para a contratação de trabalhadores mais velhos, a acomodação, a dificuldade em lidar com novas tecnologias e em se manter atualizado. 
Os resultados deste estudo podem subsidiar discussões entre gestores de instituições públicas e privadas de diversas áreas, e contribuir para que estas instituições se preparem para o envelhecimento de seus colaboradores e desenvolva programas de inserção e de preparação da aposentadoria para os profissionais mais velhos. O desenvolvimento de programas de preparação para a aposentadoria poderá favorecer um envelhecimento saudável e digno aos trabalhadores mais velhos.

Sugere-se a realização novos estudos para comparar com os resultados aqui obtidos e explorar outras estratégias inovadoras adotadas por instituições públicas e privadas que direcionem o trabalhador mais velho no seu processo de aposentadoria.

\section{Referências}

Camarano, A. A. (2016). Empregabilidade do trabalhador mais velho e reforma da previdência.

Cepellos, V. M., \& Tonelli, M. J. (2017). Envelhecimento profissional: percepções e práticas de gestão da idade. Revista Alcance (Online), 24(1), 4.

da Rocha Siqueira-Brito, A., França, L. H. F. P., \& Valentini, F. (2016). Análise fatorial confirmatória da Escala de Ageismo no Contexto Organizacional. Avaliação Psicológica, 15(3), 337-345.

de Lacerda Araújo, J., Belo, R. P., \& Resende, J. W. R. (2016). Trabalho e envelhecimento na contemporaneidade: uma análise acerca da representação social da aposentadoria. Perspectivas em Psicologia, 20(1).

de Oliveira Cavalcante, K., \& da Silva, A. B. (2017). As Implicações da capacitação nas crenças de AutoeficácIa de Gestores técnico-Administrativos de uma Instituição federal de educação superior. Revista de Administração da Universidade Federal de Santa Maria, 10(3), $474-492$.

de Oliveira Teixeira, S. M., de Souza, L. E. C., \& Maia, L. M. (2018). Ageísmo institucionalizado: uma revisão teórica. Revista Kairós: Gerontologia, 21(3), 129-149.

de Oliveira Teixeira, S. M., Marinho, F. X. S., Vasconcelos, A. M. C., \& de Oliveira Martins, J. C. (2016). Da velhice estigmatizada à dignidade na existência madura: novas perspectivas do envelhecer na contemporaneidade. Estudos e Pesquisas em Psicologia, 16(2), $469-487$.

França, L. H. D. F. P., Siqueira-Brito, A. D. R., Valentini, F., Vasques-Menezes, I., \& Torres, C. V. (2017). Ageismo no contexto organizacional: a percepção de trabalhadores brasileiros. Revista Brasileira de Geriatria e Gerontologia, 20(6), 762-772.

França, L. H. F., Nalin, C. P., Brito, A. D. R. S., Amorim, S. M., Rangel, T., \& Ekman, N. C. (2014). A percepção dos gestores brasileiros sobre os programas de preparação para a aposentadoria. Estudos interdisciplinares sobre o envelhecimento, 19(3).

Marcial, E. C., Curado, M. P. F., Oliveira, M. G. D., Cruz Júnior, S. C. D., \& Couto, L. F. (2017). Brasil 2035: cenários para o desenvolvimento.

Paolini, K. S. (2016). Desafios da inclusão do idoso no mercado de trabalho. Rev Bras Med Trab, 14(2), $177-82$.

Pazzim, T. A., \& Marin, A. (2016). Programa de Preparación para la Jubilación: Una revisión sistemática de la literatura nacional. Revista Brasileira de Orientação Profissional, 17(1), 91-101.

Pereira, A. S., Shitsuka, D. M., Parreira, F. J., \& Shitsuka, R. (2018). Metodologia da pesquisa científica. 3-9. UFSM. https://repositorio. ufsm. br/bitstream/handle/1/15824/Lic_Computacao_Metodologia-Pesquisa-Cientifica. pdf.

Raposo, M. A. M., Leite, F. M. C., \& Maciel, P. M. A. (2018). Concepção de velhice: um estudo com profissionais de saúde de um Hospital Universitário. Rev. pesqui. cuid. fundam. (Online), 958-963.

Rego, A., Vitória, A., Cunha, M. P. E., Tupinambá, A., \& Leal, S. (2017). Developing and validating an instrument for measuring managers’ attitudes toward older workers. The International Journal of Human Resource Management, 28(13), 1866-1899.

Rocha, L. S., Pelzer, M. T., Abreu, D. P. G., Rodrigues, A. P., Seiffert, M. A., \& Palmeiras, G. D. B. (2018). Idoso no mercado de trabalho: implicações para a enfermagemgerontológica. Rev. enferm. UFSM, 1-11.

Sá, R. A. D., \& Wanderbroocke, A. C. N. (2016). Significados do envelhecimento para servidoras públicas de uma instituição de ensino superior. BoletimAcademia Paulista de Psicologia, 36(90), 192-208.

Sato, A. T., Barros, J. D. O., Jardim, T. D. A., Ratier, A. P. P., \& Lancman, S. (2017). The aging process and work: a case study in the maintenance engineering division of a public hospital in the city of São Paulo, Brazil. Cadernos de saude publica.

Silva, A., \& Dal Prá, K. R. (2014). Envelhecimento populacional no Brasil: elementos para pensar o lugar das familias na proteção dos idosos. Argumentum, 6(1), 99-115.

Universidade Estadual de Londrina. Conhecendo a Universidade Estadual de Londrina. <http://portal.uel.br/conheca-a-uel/> .

Zaniboni, S. (2015). The interaction between older workers' personal resources and perceived age discrimination affects the desired retirement age and the expected adjustment. Work, Aging and Retirement, 1(3), 266-273. 